\title{
CLINICAL RESEARCH ARTICLE Perinatal nutrition impacts on the functional development of the visual tract in infants
}

\author{
Jonna Normia ${ }^{1}$, Katri Niinivirta-Joutsa ${ }^{2}$, Erika Isolauri ${ }^{1}$, Satu K. Jääskeläinen ${ }^{2}$ and Kirsi Laitinen ${ }^{3}$
}

\begin{abstract}
BACKGROUND AND OBJECTIVE: We investigated the associations of maternal diet and serum fatty acids during pregnancy and in early infancy on infantile neurodevelopment.

METHODS: Pattern-reversal visual evoked potentials ( $p V E P$ ) as depictors of central nervous system maturation were recorded from 56 children when they were 2 years old. Maternal nutrient intakes were calculated from food diaries and fish consumption from questionnaires collected during pregnancy. Serum phospholipid fatty acids were determined by gas chromatography in late pregnancy and from infants at 1 month of age.

RESULTS: The children of the women who consumed fish three or more times per week during the last trimester of pregnancy had a higher pVEP component P100 amplitude for $60^{\prime}$ (mean 23.4, SD 8.1) and 30' (mean 20.4, SD 6.7) of arcminute check sizes compared to those who consumed fish 0-2 times per week (mean 15.0, SD 4.8, $p=0.023$, adjusted for birth weight and gender $p=$ 0.058 and mean 13.4, SD 2.0, respectively, $p=0.028$, adjusted $p=0.072$ ). Maternal and child serum phospholipid fatty acids correlated with child pVEP measurements.

CONCLUSION: The results of this small-scale study suggest that fish consumption during pregnancy and perinatal serum fatty acid status may associate with neurodevelopment within visual system during infancy.
\end{abstract}

Pediatric Research (2019) 85:72-78; https://doi.org/10.1038/s41390-018-0161-2

\section{INTRODUCTION}

There is an ever-increasing numbers of patients with diet-related chronic diseases in industrialized societies, with a significant propagation in the paediatric population. The developmental origins of health and disease concept proposes that their roots lie in critical stages of development when the crucial regulatory systems are plastic and sensitive to the environment ${ }^{1}$. It has become evident from experimental and epidemiological studies that the nutritional environment in utero, as well as during the neonatal period exerts significant effects on the risk of an individual developing non-communicable diseases. Many nutrients have been linked directly or through metabolic routes such as their interaction with gut microbiota, to infantile neurological development, thus highlighting the importance of the nutritiongut-brain axis as a modulator of health and development ${ }^{2-4}$.

Maternal diet is a modifiable environmental component with the possibility to promote foetal development to its optimal potential. The supply of long chain polyunsaturated fatty acids (LC-PUFA) is of particular interest regarding neurological development as these are important components of neural and retinal membranes ${ }^{5}$. At present, at odds with the recommendations, the dietary intake of fats is typically becoming shifted towards a higher intake of saturated fatty acids at the expense of unsaturated fatty acids, combined with a low intake of n-3 fatty acids. The importance of adequate supply of these fatty acids culminates during the third trimester of pregnancy as well as in the first months of life, when considerable quantities of LC-PUFA, especially docosahexaenoic acid (DHA), accumulate in the brain and retina ${ }^{6}$. Although many important maturation processes extend beyond infancy, most studies have utilized some form of neurophysiologic assessment of the visual system as an indicator of neurodevelopment; these tend to have focused on early infancy, and up to one year of age, and on either maternal or infant nutrition, although there are some studies which have also evaluated children's development over longer periods ${ }^{7,8}$.

We studied here the influence of nutritional LC-PUFA sources both in the maternal diet and serum as well as in their children's diet and serum on the infants' neurodevelopment as reflected in visual system function at the age of two years from a larger mother-child study aiming to modify maternal dietary intake to improve the health of both the mother and child ${ }^{9,10}$. For this purpose, we chose to use pattern-reversal visual evoked potentials (pVEP), a sensitive and accurate, non-invasive method that can be used to assess visual function and maturational changes occurring within the visual pathways during the first years of life. The aim of this study was to investigate firstly whether the mother's diet and fatty acid status during pregnancy or the child's fatty acid status at one month of age would exert any impact on the infant's neurodevelopment and visual system function at 2 years of age by undertaking pVEP recordings with two check sizes, 30 and 60 arch minutes. The second aim was practical: to gather laboratory specific reference values for pVEP recordings in small children.

\section{METHODS}

Study subjects and design

We performed pVEP recordings in 2-year-old children participating in a mother-child intervention study; its primary focus was to

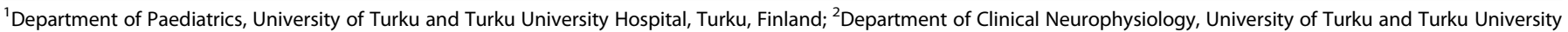
Hospital, Turku, Finland and ${ }^{3}$ Institute of Biomedicine, University of Turku, Turku, Finland

Correspondence: Kirsi Laitinen (kirsi.laitinen@utu.fi)

Received: 20 December 2017 Revised: 18 July 2018 Accepted: 23 July 2018

Published online: 20 September 2018 
define the impact of maternal diet and serum phospholipid fatty acids during pregnancy on the child's neurodevelopment as reflected in visual system function. The original intervention setting of the clinical trial has been described previously (9; NCT00167000). Briefly, it involved randomization of the mothers into three study groups: diet counselling with or without probiotics $\left(1 \times 10^{10}\right.$ colony-forming units of Lactobacillus rhamnosus GG, ATCC 53103, Valio Ltd, Helsinki, Finland and Bifidobacterium lactis Bb-12, Chr. Hansen, Hoersholm, Denmark each), or a control group receiving placebo at the first trimester of pregnancy until the end of exclusive duration of breastfeeding maximum of six months after delivery. The diet counselling aimed at a dietary intake complying with that recommended for pregnant women ${ }^{11}$.

The VEP investigation at the child's age of 2 years was completed to a convenience sample of children whose parents were willing, and the children were born at term, and who had no clinically evident visual disorder (poor sight/squint) or epilepsy. VEP measurements were performed at the age of 2, because the process of myelination should be complete by the age of two years. VEP recording took about 30 to $45 \mathrm{~min}$ including preparation. Of the 82 investigations, 56 were successfully completed. The subject flow is shown in Fig. 1.

Dietary intake was collected from mothers during each trimester of their pregnancy and from their children at the age of 2 years. Serum phospholipid fatty acid composition and concentration were measured in the third trimester of pregnancy from mothers and at the age of 1 month from the children. Demographic and clinical information were obtained by interview or from health care records. Heights and weights of the infants were measured by a research nurse using standard methods.

The Ethical Committee of the Hospital District of Southwest Finland approved the study protocol and the mothers provided written informed consent. The study complies with the Declaration of Helsinki as revised in 2000.

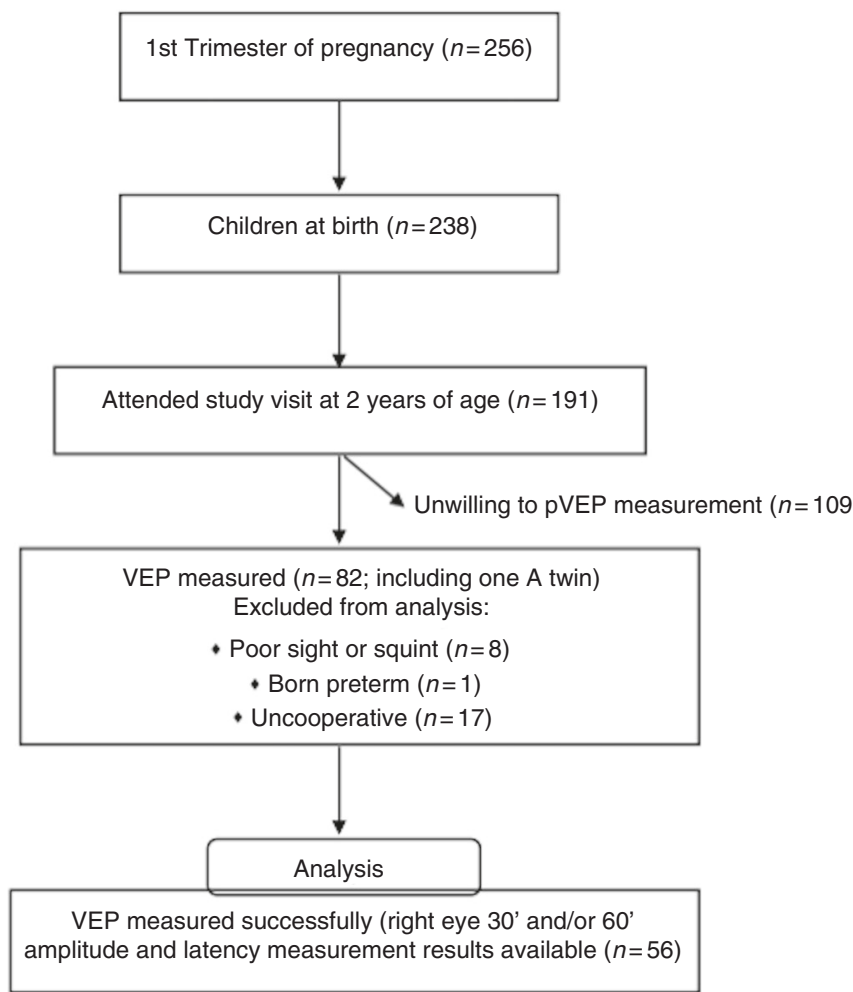

Fig. 1 Flow chart. Reasons for discontinuation: miscarriage, illness in mother or child, moving, not willing to continue
Analyses of diet and serum fatty acids

Dietary intake was recorded in 3-day food diaries, including one weekend day, in each trimester of pregnancy. The women received personal and written instructions on how to fill in the food diaries and the diaries were checked for completeness and accuracy during the study visits. Mean daily intake of nutrients over pregnancy was calculated using a computerized programme (Micro-Nutrica ${ }^{\circledR}$, version 2.5, Research Centre of the Social Insurance Institution, Turku, Finland). The frequency of consuming fish over the previous two weeks prior to the third trimester of pregnancy study visit was inquired by a questionnaire.

Serum phospholipid fatty acid analyses were conducted as previously described ${ }^{12,13}$. Briefly, total lipids were extracted from blood samples with chloroform:methanol and phospholipids were separated by solid phase extraction. Fatty acid methyl esters were prepared with boron trifluoride $\left(\mathrm{BF}_{3}\right)$ using the methanol procedure and fatty acids were analysed by gas chromatography.

Visual evoked potentials

The visual system was assessed with pVEPs recorded with an eight-channel Viking IV ENMG and evoked potential device (Nicolet Biomedical Instruments, Madison, Wisconsin). Three bipolar derivations of $\mathrm{O} 1, \mathrm{Oz}$ and $\mathrm{O} 2$ electroencephalogram (EEG) electrodes referenced to the $\mathrm{Fpz}$ electrode were used according to the international 10-20 EEG electrode placement system. A ground electrode was placed on the forehead. Scalpelectrode impedance was kept below $5 \mathrm{kOhm}$.

Visual stimuli were generated with Medelec ST 10 Sensor (Oxford Instruments, Old Woking, UK) and consisted of a high contrast black-and-white checkerboard generated on a Sony monitor. The mean luminance of the screen was $100 \mathrm{~cd} / \mathrm{m}^{2}$, and the contrast between the black-and-white squares was greater than $90 \%$. The checkerboard pattern was reversed at a rate of $2.3 \mathrm{~Hz}$.

The evoked EEG signals were amplified and bandpass-filtered at $0.5-200 \mathrm{~Hz}$ (sampling rate, $5 \mathrm{Hkz}$ ). At least one hundred pVEPs time-locked to the stimulus were averaged per trial, which was repeated at least two times to ensure consistency of the waveforms.

Recordings were done only when the child was alert and attentive. The child was seated on the parent's lap $70 \mathrm{~cm}$ away from and at the level of the stimulus screen. The tester (J.N.) attracted the child's attention towards the screen with small figure stickers kept at the fixation point in the middle of the screen. The mother and the tester continuously monitored the child's visual fixation to the stimulus, and the recording was temporarily discontinued when the child was not fixating or moved nearer or further from the screen. Thus, the computer only acquired data during those periods when the child was viewing the stimulus. The recording was always done first to the right eye (with an eyepatch placed on the other side) and then to the left eye if cooperation allowed. The first stimulus presented to the child was a 60 arcminute pattern-reversal checkerboard. If there was a clearly reproducible $\mathrm{pVEP}$, also pVEP with smaller 30 arcminute checks were tested. The main characteristics of the pattern-reversal VEP waveform investigated were the peak latencies (N75 and P100) and amplitudes (P100) of the main components. All pVEP records were scored by the same experienced electrophysiologist (S.J.), who was unaware of the group assignment.

Statistical analysis

The primary endpoint variables were measured from the midline Oz electrode to the stimulation of the right eye and consisted of the latency and amplitude of the P100 components for each chequerboard size, $30^{\prime}$ and $60^{\prime}\left(30^{\prime}\right.$ and $60^{\prime}$ latency, and $30^{\prime}$ and $60^{\prime}$ amplitude).

The correlations of potential confounders (Supplemental tables S1 and S2) with pVEP recordings were evaluated by 
Pearson correlation coefficient and Anova. Weight and head circumference at birth were found to correlate with $30^{\prime}$ latency and gender with $60^{\prime}$ amplitude (Supplemental tables S1 and S2). All the analyses were adusted for birth weight and gender. Head circumference was not used for adjusting as it correlated with birth weight. The intervention effect of the original clinical trial setting and the VEP measurements in the groups with different frequencies of fish consumption per week were compared by Anova and by adjusted Anova. The relationships between diet and serum phospholipid fatty acids with pVEP recordings were evaluated by searching for correlations using Spearman partial correlation coefficients. In the evaluation of the reference values of the PVEP variables, their normality was first tested by the Kolmogorov-Smirnov test. The 2.5 and $97.5 \%$ percentiles were defined as mean-1.96*SD and mean $+1.96 *$ SD. Statistical analysis was performed using IBM SPSS Statistics for Windows (version 23.0, Armonk, NY, IBM Corp). P-values less than 0.05 were considered statistically significant.

\section{RESULTS}

Clinical characteristics

The primary outcome variables of the pVEP recordings were available for 56 children (Fig. 1) recorded at the mean age of 2.1 years (SD 0.2 years). Except for one child investigated at the age of 3.2 years, all children underwent the pVEP recording at the age of 2.0 to 2.3 years $(n=55)$. The clinical characteristics of the children at birth and at 2 years of age are shown in Table 1. The growth of the children was within the normal reference range, but four children were tall for their age (height SD score above two) at the age of 2 years. The children were considered healthy, although one child had situs inversus at birth.

The mothers were 28.9 (SD 4.6) years of age and they were of normal weight as evaluated by their mean BMI $(23.8$, SD 3.8) prior to pregnancy, although $32 \%$ were overweight. The weight gain during pregnancy was on average 15.3 (4.9 SD) kg. The mothers were normotensive and normoglycemic: mean systolic blood

\begin{tabular}{|c|c|c|}
\hline Characteristics & $\mathrm{N}(\%)$ & Mean (SD) \\
\hline \multicolumn{3}{|l|}{ At birth } \\
\hline Male gender & $28(50)$ & \\
\hline Gestational age, weeks & & $39.5(1.4)$ \\
\hline Weight, $\mathrm{g}$ & & $3579(486)$ \\
\hline Weight for height, \% & & $-2.0(9.7)$ \\
\hline Length, $\mathrm{cm}$ & & $51(1.8)$ \\
\hline Length, SD & & $0.40(1.0)$ \\
\hline Head circumference, $\mathrm{cm}$ & & $34.9(1.3)$ \\
\hline Head circumference, SD & & $-0.1(0.9)$ \\
\hline Caesarean delivery $^{a}$ & 10(18) & \\
\hline Vaginal delivery ${ }^{\mathrm{a}}$ & $45(82)$ & \\
\hline \multicolumn{3}{|l|}{ At 2 years of age } \\
\hline Weight, kg & & $12.7(1.3)$ \\
\hline Weight for height, $\%$ & & $-1.9(7.4)$ \\
\hline Height, $\mathrm{cm}$ & & $88.0(3.2)$ \\
\hline Height, SD & & $0.1(1.0)$ \\
\hline \multicolumn{3}{|l|}{ Duration of breastfeeding } \\
\hline Exclusive, weeks & & $13.8(6.7)$ \\
\hline Total, weeks & & $41.2(23.4)$ \\
\hline
\end{tabular}

pressure during the pregnancy was 114 (SD 9) $\mathrm{mmHg}$, diastolic blood pressure was 68 (SD 6) $\mathrm{mmHg}$ (mean of three measurements during the pregnancy), and at the group level, the mean fasting blood glucose was 4.6 (SD 0.3 ) $\mathrm{mmol} / \mathrm{l}$ (mean of two measurements during the pregnancy; early and late pregnancy). Gestational diabetes was diagnosed in 14 out of 52 women (data missing from 4 mothers) in prenatal welfare clinics. A total of 19 of the women (34\%) had smoked before pregnancy. Dietary intakes of mothers during pregnancy and children at the age of 2 years are presented in Table 2.

pVEP recording results

The results for the right and left eye pVEP component P100 latencies and amplitudes with $30^{\prime}$ and $60^{\prime}$ check sizes are presented in Table 3. The right and left eye measurements correlated as follows: $60^{\prime}$ latency, $r=0.475, p=0.005 ; 30^{\prime}$ latency $r=0.355 . p 0.059 ; 60^{\prime}$ amplitude $r=0.746, p<0.001,30^{\prime}$ amplitude $0.715, p<0.001$. Mother's BMI or overweight status (yes/no) prior to pregnancy, gestational weight gain, blood glucose levels, presence of gestational diabetes (yes/no), systolic or diastolic blood pressure or smoking prior to pregnancy (yes/no) were not associated with the results of their child's pVEP recordings (all NS, Pearson correlation coefficient test or Anova; Supplemental tables S1 and S2). The child's gestational age, duration of breastfeeding (total or partial), birth height or child's age at pVEP recording were not correlated with pVEP recording results (all NS, Supplemental table S1). Weight and head circumference at birth correlated with

Table 2. Dietary intake and serum phospholipid fatty acids of the mothers and their children

\begin{tabular}{|c|c|c|c|c|}
\hline & $\begin{array}{l}\text { Mothers } \\
\text { mean }\end{array}$ & SD & $\begin{array}{l}\text { Children } \\
\text { mean }\end{array}$ & SD \\
\hline Diet intake ${ }^{a}$ & $(N=56)$ & & $(N=53)$ & \\
\hline Energy (kcal) & 2041 & 354 & 1042 & 216 \\
\hline Protein (\% of energy) & 16.2 & 2.0 & 18.0 & 3.0 \\
\hline Carbohydrates (\% energy) & 51.2 & 4.9 & 52.1 & 4.9 \\
\hline Total fat (\% of energy) & 31.2 & 4.6 & 28.6 & 5.0 \\
\hline SFA (\% of energy) & 12.8 & 2.2 & 11.8 & 3.2 \\
\hline MUFA (\% of energy) & 10.8 & 1.9 & 9.6 & 2.2 \\
\hline PUFA (\% of energy) & 5.5 & 1.0 & 4.4 & 1.4 \\
\hline Vitamin D $(\mu \mathrm{g})$ & 9.4 & 3.7 & 4.7 & 2.6 \\
\hline Vitamin E (mg) & 14.6 & 5.0 & 4.8 & 2.1 \\
\hline $\begin{array}{l}\text { Fatty acids (\% of total fatty } \\
\text { acids) }^{b}\end{array}$ & $(N=20)$ & & $(N=15)$ & \\
\hline 18:2n-6 (linoleic acid) & 19.4 & 2.2 & 18.5 & 2.0 \\
\hline $20: 4 n-6$ (arachidonic acid) & 7.2 & 1.0 & 9.5 & 1.8 \\
\hline Sum n-6 & 30.6 & 2.3 & 31.7 & 1.4 \\
\hline $\begin{array}{l}\text { 18:3n-3 (alpha-linolenic } \\
\text { acid) }\end{array}$ & 0.6 & 0.2 & 0.2 & 0.04 \\
\hline $\begin{array}{l}\text { 20:5n-3 (eicosapentaenoic } \\
\text { acid) }\end{array}$ & 1.6 & 0.9 & 0.7 & 0.3 \\
\hline $\begin{array}{l}\text { 22:6n-3 (docosahexaenoic } \\
\text { acid) }\end{array}$ & 4.9 & 1.1 & 4.0 & 0.9 \\
\hline Sum n-3 & 7.7 & 2.0 & 5.6 & 1.2 \\
\hline SFA & 44.9 & 0.8 & 45.2 & 1.7 \\
\hline MUFA & 16.2 & 1.3 & 16.9 & 2.6 \\
\hline PUFA & 38.9 & 1.7 & 37.8 & 2.1 \\
\hline
\end{tabular}




\begin{tabular}{|c|c|c|c|c|c|c|c|c|c|c|c|}
\hline & $N$ & Mean & Md & SD & Min & Max & \multicolumn{5}{|c|}{ Percentiles } \\
\hline \multicolumn{12}{|l|}{ Right eye } \\
\hline $60^{\prime}$ latency & 55 & 108.2 & 109.0 & 4.1 & 95.5 & 117.0 & 97.7 & 105.0 & 109.0 & 111.0 & 116.2 \\
\hline $30^{\prime}$ amplitude & 43 & 17.4 & 13.8 & 8.5 & 4.1 & 40.0 & 4.4 & 12.3 & 13.8 & 23.0 & 39.6 \\
\hline \multicolumn{12}{|l|}{ Left eye } \\
\hline $60^{\prime}$ latency & 34 & 108.2 & 109.0 & 4.0 & 97.5 & 116.0 & 100.3 & 105.8 & 109.0 & 111.0 & 116.1 \\
\hline $30^{\prime}$ latency & 30 & 110.3 & 110.5 & 6.5 & 85.0 & 120.0 & 97.5 & 109.0 & 110.5 & 115.0 & 123.0 \\
\hline $60^{\prime}$ amplitude & 34 & 20.2 & 19.0 & 8.5 & 7.7 & 44.0 & 3.6 & 14.4 & 19.0 & 24.3 & 36.8 \\
\hline
\end{tabular}

30 latency $(r=0.340, p=0.025$ and $r=0.414, p=0.006$, respectively) but not with other pVEP recording results. Gender was related to $60^{\prime}$ amplitude $(p=0.009)$. The further analyses were completed adjusted for weight at birth and gender.

Impact of maternal diet and serum fatty acids on the child's pVEP recording results. The dietary intervention with or without probiotic supplementation of the original clinical trial study setting was not statistically significantly associated with the pVEP latencies or amplitudes (NS, ANOVA, Supplemental table S3). Thus, in the subsequent analyses, the study population was examined as a single group.

The women consumed on average 34 (SD 40) g of fish daily $(n=19)$, the median frequency of consumption being three times per week (range 0 to 6 times per week). The median value of fish consumption was used as a cut-off in the analysis for evaluation the impact on child visual development. The children of the women who consumed fish three or more times per week during the last trimester of pregnancy had a higher right eye $60^{\prime}$ amplitude (mean 23.4, SD $8.1 \mu \mathrm{V}$ ) and $30^{\prime}$ amplitude (mean 20.4, SD $6.7 \mu \mathrm{V}$ ) compared to those who consumed fish 0 to 2 times per week (60' amplitude mean 15.0, SD $4.8 \mu \mathrm{V}, p=0.023$, adjusted $p=0.058 ; 30^{\prime}$ amplitude mean 13.4, SD $2.0 \mu \mathrm{V}, p=0.028$, adjusted $p=0.072$, independent samples $t$-test). No differences in pVEP latencies were observed regarding the mothers' fish intake.

Maternal dietary intake of saturated fatty acids (SFA) during pregnancy was negatively correlated with right eye $30^{\prime}$ latency $(\rho=-0.311$, adjusted $p=0.048)$, but no associations were detected with $60^{\prime}$ latency or VEP amplitudes (Supplemental table S4). Maternal dietary intakes of energy, monounsaturated fatty acids (MUFA), PUFA, protein or carbohydrate, or intakes of vitamin $D$ or $E$ were not associated with any of the PVEP variables (all NS, Supplemental table S4).

The proportion of alpha-linolenic acid (C18:3n-3) in maternal serum phospholipids at the third trimester of pregnancy was correlated with longer $30^{\prime}$ latencies in the children $(\rho=0.637$, adjusted $p=0.019$ ). No other correlations between maternal fatty acids and children's pVEP recording results were seen, as evaluated by the proportions of the phospholipid fatty acids (Supplemental table S5). In the quantitative evaluation of the serum phospholipid fatty acids, the maternal alpha-linolenic acid concentration correlated with a longer $30^{\prime}$ latency $(\rho=0.578$, adjusted $p=0.038)$ and a higher $30^{\prime}$ amplitude $(\rho=0.624$, adjusted $p=0.023)$. Furthermore, higher linoleic acid $(18: 2 n-6)$ concentration $(\rho=0.650$, adjusted $p=0.016)$ and sum on $n-6$ FA $(\rho=0.558$, adjusted $p=0.048)$, higher total SFA $(\rho=0.701$, adjusted $p=0.008)$ and total PUFA $(\rho=0.574$, adjusted $p=0.040$ ), were associated with a higher $30^{\prime}$ amplitude in the infants (Supplemental table S5).

Impact of the child's diet and serum fatty acids on the pVEP recording results. When evaluating the infants' serum phospholipid fatty acids at 1 month of age in relation to 2-year-VEP measurements, a positive correlation was found between the proportion of arachidonic acid (20:4 n-6; $\rho=0.664$, adjusted $p=$ $0.013)$, DHA (22:6n-3; $\rho=0.582$, adjusted $p=0.037)$ and sum of n- 6 fatty acids $(\rho=0.595$, adjusted $p=0.032)$ with a longer $60^{\prime}$ latency (Supplemental table S6). The children's dietary intake of energy was negatively correlated with $30^{\prime}$ amplitude $(\rho=-0.319$, adjusted $p=0.046$ ), whilst total fat, SFA, MUFA and PUFA, protein and carbohydrates or fat soluble vitamins $D$ and $E$ at 2 years of age were not correlated with any of the right eye pVEP variables (all NS, Supplemental table 4).

Pattern VEP reference values for 2-year-old children. Reference values for 2-year-old children were calculated from the right eye and left eye recordings (Table 3 ). There were no statistically significant differences between the right and left eye pVEPs. The pVEP variables were normally distributed and no differences were detected between girls and boys, except that right eye $60^{\prime}$ amplitudes were higher in girls than in boys $(21.5$, SD $8.1 \mu \mathrm{V}$ vs 16.4 , SD $5.7 \mu \mathrm{V}, p=0.009$ ). Despite this difference in one variable, common reference values for girls and boys were calculated due to the relatively small number of children in whom we acquired reliable pVEP recordings.

\section{DISCUSSION}

We have demonstrated here that even as early as the foetal period, the nutritional quality may be of significance in the child's neurodevelopment when this is assessed via the function of the visual system at 2 years of age. In particular, the maternal intake of fish for at least three times per week was related to an increase in right eye $60^{\prime}$ amplitude (statistically non-significant after adjusting for confounding factors), and a lower intake of SFA was related to a decrease in right eye $30^{\prime}$ min latency. These observations were further substantiated by evaluations of the serum phospholipid fatty acid status. It is of note that the study was conducted with a small number of participants and the results need to be verified in a larger setting using the robust pVEP recording for evaluation of the child development. These associations point to a fascinating opportunity for shaping the myelination of the visual tracts (a decrease in latency) and synaptogenesis, which in turn would impact on synaptic efficiency and degree of synchrony of the impulses travelling within the visual pathway (i.e., causing an 
increase in amplitude) by optimizing the dietary fatty acid intake during pregnancy.

The results on child serum fatty acids in relation to later pVEP measurements may be taken as supporting the results obtained for maternal diet and fatty acids status, except for a surprising relation of $\mathrm{DHA}$ intake with a longer $60^{\prime}$ latency. The relation of fatty acids with child development has been indicated previously, like in a study where the concentration of red blood cell DHA at the age of four months was related to mental development index (Bayley Scales of Infant Development) at the age of 18 months ${ }^{14}$. It is noteworthy that the children in our study were breastfed at the time of blood sampling at one month of age and thus the maternal diet is a clear contributor to the child's fatty acid status $^{12,13}$. It would be important to conduct more studies focusing on this specific time frame in early infancy in order to evaluate in detail which type of diet in infancy would be most beneficial regarding the child's later neurodevelopment. There is one systematic review of LC-PUFA supplementation of breastfeeding mothers but it could not provide any evidence on whether it conferred benefits to the child's development as evaluated by various outcomes including language development, solving problems, psychomotor development or visual acuity ${ }^{15}$. The benefit of breastfeeding over formula feeding of infants for neural and visual maturation has been indicated ${ }^{16,17}$. Interestingly, maternal dietary patterns with poor food choices have been previously associated with lower scores in cognitive and psychomotor development tests in preschool aged children ${ }^{18}$. One option for infants needing formula feeding is supplementation of the infant formula with DHA and arachidonic acid that was shown to result in better visual acuity using sweep visual evoked potential compared to infants fed formula without these LCPUFAs ${ }^{19,20}$, although contradictory results exists ${ }^{16}$. Nevertheless, a sufficient supply of LC-PUFAs is likely to be of importance upon weaning from the breast milk ${ }^{21}$

The published human studies provide contradictory data regarding maternal diet or fatty acid status during pregnancy on child development even though variable methods have been used to evaluate this phenomenon (reviewed in ref. ${ }^{22}$ ). Generally, an increase in n-3 fatty acids either through consumption of relevant food sources, mainly fish or supplement use, has been considered as being beneficial in view of the importance of $n-3$ LC-PUFAs as structural and functional components in brain and retina ${ }^{23}$. The results of our study suggests that frequent fish consumption is of benefit for child development that may be attributable to LC-PUFAs within fish, but also due to other nutrients like vitamin $D$ and $E$, also import for development ${ }^{24,25}$. In addition to n-3 LC-PUFAs, n-6 fatty acids are incorporated into brain and are needed for infant growth and development ${ }^{26}$, which was also indicated in our study as infants' $n-6$ LC-PUFA arachidonic acid and sum of $n-6$ fatty acids was related to longer $60^{\prime}$ latencies. Nevertheless, the impact of $n-6$ LC-PUFAs on neurodevelopment need to be established ${ }^{27}$. One example that demonstrates the challenges in establishing the impact of perinatal nutrition on child neurodevelopment may be taken from a recent study ${ }^{28}$ that could not demonstrate either benefit or harm by a combination of supplementation of a n-3 LC-PUFA and $n-6$ fatty acid (arachidonic acid) balanced diet from the $15^{\text {th }}$ week of gestation to four months postpartum on infantile neurodevelopment at the age of 4-5 years.

The difficulty in demonstrating the benefits of perinatal nutritional impact on child neurodevelopment may arise from various sources. Firstly, the evaluation of the habitual maternal diet in a robust way is time-consuming and laborious both to the researchers and to the study participants. In our study, we used a repeated food diary analysis, conducted three times over the pregnancy to increase the reliability of the dietary intake of nutrients and we also used a frequency questionnaire for the longer-term evaluation of fish intake, which may be considered an appropriate means for the assessment of foods that are not consumed daily. In our study, we applied a dietary approach which is reasonable in the public health care setting. We demonstrated previously in a larger number of subjects of the same study that dietary intake of pregnant women may be modified, particularly the intake of SFA lowered and the intake of PUFA increased, by dietary counselling ${ }^{11}$, which could also contribute to the child development, although as demonstrated in this small-scale study, the intervention group allocation per se did not influence the child development. Previously, several supplement studies have been completed (including ref. ${ }^{29}$ ); their benefits lie in their ability to increase the intakes of specific n-3 fatty acids, particularly in non-fish eaters combined with the avoidance of potential harm due to pollutants in fish, although compliance in consuming the capsules may be an issue. Nevertheless, despite intensive research in the area of fatty acid supplementation, no conclusive evidence exists thus far regarding child neurodevelopment or cognition and visual outcomes (reviewed in ref. ${ }^{22,30}$ ), the benefits mainly manifested in preterm born infants ${ }^{31}$. In agreement with our study, although in that other publication, the child's development was evaluated by a psychological test, Mendez and co-workers ${ }^{32}$ found that maternal fish consumption in excess of two to three times per week was associated with higher test scores when the child was four years old. One contributor to the controversial clinical findings may be that the optimal dose of the n-3 LC-PUFAs (particularly DHA) and the ratio of $n-3$ to $n-6$ fatty acids for child's neurodevelopment remains to be defined. Equally provision from the diet and their ratio of the precursors for DHA and arachidonic acid, namely $n-6$ fatty acid linoleic acid and $n-3$ fatty acid alpha-linolenic acid may be of importance for the conversion to the longer chain fatty acids, although this was shown not to have an impact on VEP acuity ${ }^{33}$. Along with considering the optimal dietary intake, the transfer from mother to foetus of fatty acids may be limited in cases of compromised pregnancies, e.g., in diabetic mothers ${ }^{34}$. In healthy mothers, there is a preferential transfer of LC-PUFA over other fatty acids through placenta ${ }^{35}$. This is of importance as fetuses and infants have a limited capacity to synthesize $n-3$ LC-PUFA (reviewed in ref. ${ }^{22}$ ). Maternal genetic variability may be a contributor to the maternal LC-PUFA status, but no evidence was revealed of its impact on the child's cognitive development as evaluated by Bayley Scales of Infant Development II test in a longitudinal observational study in high fish consumers at the age of 20-30 months ${ }^{36}$. In addition, breastfeeding or formula feeding status of the infant may interfere with defining the effect of pregnancy on the child's neurodevelopment ${ }^{37}$. We found no relation between the maternal background factors like prepregnancy overweight or smoking status or presence of gestational diabetes regarding the child's visual system function, but some potential effect may not be ruled out as our study population was small. A previous study in 185 infants demonstrated that birth weight, gender and number of smokers in the household influence VEP acuity and the authors speculated that a range of perinatal factors could either mask or enhance the dietary effects on child development ${ }^{38}$. In our study we found that weight and head circumference at birth and gender correlated with pVEP recordings and consequently the results were adjusted.

Another likely cause for differences in the study results may arise from the methods, particularly those used to evaluate neurodevelopment. Several studies have focused on the evaluation of perinatal diet on infantile mental performance and psychomotor development instead of neurodevelopment ${ }^{22}$, which was the focus in the present study using eye pVEP measurement known to be a robust test. Many previous studies investigating relationships between nutritional factors and neurodevelopment have exploited either electroretinography that 
reflects the function of the retinal cells, not the higher level central nervous system (CNS) maturation, or sweep-VEP giving a crude measure of visual acuity in babies (reviewed in ref. ${ }^{39}$ ). As a surrogate for optic nerve and CNS function, pVEP allows a detailed investigation of the components contributing to CNS maturation. The latency of the pVEP reflects the myelination of the visual tracts, being shorter when there is a higher degree of myelination. The waveform and amplitude of the pVEP response are associated with synaptogenesis, synaptic efficiency and degree of synchrony of the impulses travelling within the visual pathway. Furthermore, by applying different chequerboard sizes (e.g., 60 and 30 arch minutes), it is possible to conduct an objective and detailed analysis of the maturational state of visual acuity. Thus, pVEP recordings enable quantifying these aspects of neurodevelopment and visual function, already at two years of age when children are old enough to permit successful recordings. Furthermore, we were able to collect valid and feasible pVEP reference values, which have been successfully applied to clinical diagnostics in small children with visual problems. However, a larger population would have been needed in order to define reference values separately for boys and girls.

In addition to the robust methods used for the evaluation of child neurodevelopment, the carefully collected clinical trial data and the long-term follow-up with good compliance may be viewed as a strength of our study. At the same time, considering the long duration of the follow-up, a larger study would be required in order to evaluate the impact of any individual dietary intervention. As evaluated from our data about 240 subjects per group would be needed to produce statistically significant differences in ocular pVEPs in a similar study setting. Our approach may be recommended, taking into consideration both the comprehensive maternal dietary composition and the impact of gut microbiota on neurodevelopment of the child. We consider our study population to be representative of a typical group of women attending the communal maternal health care system, which is available to all women free-of-charge in Finland, with the women consuming a typical diet for the region. Our population was thus well-nourished and healthy and the children were born at term. Many of the previous studies have focused on undernourished populations or on prematurity, or have aimed to modify the maternal diet by administering supplements. The nature of our study allowed the demonstration of the importance of maternal diet during pregnancy on the child's neurodevelopment that may be applicable to the majority of pregnant women; thus it provides evidence for the potential benefits of public health approaches targetted to pregnant women.

\section{CONCLUSIONS}

Our results suggest important associations between maternal diet composition during pregnancy, particularly the intake of fish, and perinatal serum fatty acid status on a child's neurodevelopment within the visual system in infancy as measured by pVEP. These results support the previous findings about the importance for child development of perinatal nutrition when permanent beneficial changes may be induced through appropriate environmental exposures. Our study also highlights the potential importance of subtle changes in the diet of healthy women with uncompromised pregnancies, beyond prematurity or nutritional deficiencies, in regulating infantile neurodevelopment. Our results may be applied for dietary counselling of pregnant women taking into account that practical advice and tools are needed for implementation of successful lifestyle changes ${ }^{40,41}$. This is of importance as the composition of the maternal diet during pregnancy and breastfeeding is the key contributor to the supply and deposition of LC-PUFAs to the foetus and infant brain ${ }^{23}$ during the period of maximum brain growth that extends throughout the first years of postnatal life.

\section{ACKNOWLEDGEMENTS}

The authors thank Tuija Poussa, Stat Consulting, and Simo Teperi, Department of Biostatistics, University of Turku, for performing the statistical analyses and Ewen MacDonald for revision of the English text. The clinical trial data collection was funded by Academy of Finland and Social Insurance Institution of Finland.

\section{ADDITIONAL INFORMATION}

The online version of this article (https://doi.org/10.1038/s41390-018-0161-2) contains supplementary material, which is available to authorized users.

Competing interests: The authors declare no competing interests.

Publisher's note: Springer Nature remains neutral with regard to jurisdictional claims in published maps and institutional affiliations.

\section{REFERENCES}

1. Hanson, M. A. \& Gluckman, P. D. Early developmental conditioning of later health and disease: physiology or pathophysiology? Physiol. Rev. 94, 1027-1076 (2014).

2. Anjos, T. et al. Nutrition and neurodevelopment in children: focus on NUTRIMENTHE project. Eur. J. Nutr. 52, 1825-1842 (2013).

3. Isolauri E., Rautava S., Collado M. C., Salminen S. Early microbe contact in defining child metabolic health and obesity risk. eds. Green L. R., Hester R. L. In Parental Obesity: Intergenerational Programming and Consequences. Physiology in Health and Disease. The American Physiological Society (Springer, New York, 2016).

4. Sharon, G., Sampson, T. R., Geschwind, D. H. \& Mazmanian, S. K. The central nervous system and the gut microbiota. Cell 167, 915-932 (2016).

5. Koletzko, B. et al. The roles of long-chain polyunsaturated fatty acids in pregnancy, lactation and infancy: review of current knowledge and consensus recommendations. J. Perinat. Med 36, 5-14 (2008).

6. Malcolm, C. A., McCulloch, D. L., Montgomery, C., Shepherd, A. \& Weaver, L. T. Maternal docosahexaenoic acid supplementation during pregnancy and visual evoked potential development in term infants: a double blind, prospective, randomised trial. Arch. Dis. Child Fetal Neonatal Ed. 88, F383-F390 (2003).

7. Hoffman, D. R. et al. Impact of early dietary intake and blood lipid composition of long-chain polyunsaturated fatty acids on later visual development. J. Ped. Gastroenterol. Nutr. 31, 540-553 (2000)

8. Uauy, R., Hoffman, D. R., Peirano, P., Birch, D. G. \& Birch, E. E. Essential fatty acids in visual and brain development. Lipids 36, 885-895 (2001).

9. Laitinen, K., Poussa, T. \& Isolauri, E. Probiotics and dietary counselling contribute to glucose regulation during and after pregnancy: a randomised controlled trial. Brit J. Nutr. 101, 1679-1687 (2009).

10. Aaltonen, J. et al. Impact of maternal diet during pregnancy and breastfeeding on infant metabolic programming: a prospective randomized controlled study. Eur. J. Clin. Nutr. 65, 10-19 (2011).

11. Piirainen, T., Isolauri, E., Lagström, H. \& Laitinen, K. Impact of dietary counselling on nutrient intake during pregnancy: a prospective cohort study. Br. J. Nutr. 96, 1095-1104 (2006).

12. Niinivirta, K., Isolauri, E., Laakso, P., Linderborg, K. \& Laitinen, K. Dietary counseling to improve fat quality during pregnancy alters maternal fat intake and infant essential fatty acid status. J. Nutr. 141, 1281-1285 (2011).

13. Hautero, U. et al. Proportions and concentrations of serum n-3 fatty acids can be increased by dietary counseling during pregnancy. Eur. J. Clin. Nutr. 67, 1163-1168 (2013).

14. Birch, E. E., Garfield, S., Hoffman, D. R., Uauy, R. \& Birch, D. G. A randomized controlled trial of early dietary supply of long-chain polyunsaturated fatty acids and mental development in term infants. Dev. Med Child Neurol. 42, 174-181 (2000).

15. Delgado-Noguera, M. F., Calvache, J. A., Bonfill Cosp, X., Kotanidou, E. P. \& GalliTsinopoulou, A. Supplementation with long chain polyunsaturated fatty acids (LCPUFA) to breastfeeding mothers for improving child growth and development. Cochrane Database Syst. Rev. 4, CD007901 (2015).

16. Makrides, M., Neumann, M. A., Simmer, K. \& Gibson, R. A. A critical appraisal of the role of dietary long-chain polyunsaturated fatty acids on neural indices of term infants: a randomized, controlled trial. Pediatrics 105, 32-38 (2000).

17. Khedr, E. M., Farghaly, W. M., Amry, S.-D. \& Osman, A. A. Neural maturation of breastfed and formula-fed infants. Acta Paediatr. 93, 734-738 (2004).

18. Leventakou, V. et al. Dietary patterns in early childhood and child cognitive and psychomotor development: the Rhea mother-child cohort study in Crete. Br. J. Nutr. 115, 1431-1437 (2016).

19. Birch, E. E. et al. Visual maturation of term infants fed long-chain polyunsaturated fatty acid-supplemented or control formula for 12 mo. Am. J. Clin. Nutr. 81, 871-879 (2005). 
Perinatal nutrition impacts on the functional development of the visual... $\mathrm{J}$ Normia et al.

78

20. Morale, S. E. et al. Duration of long-chain polyunsaturated fatty acids availability in the diet and visual acuity. Early Hum. Dev. 81, 197-203 (2005).

21. Hoffman, D. R. et al. Visual function in breast-fed term infants weaned to formula with or without long-chain polyunsaturates at 4 to 6 months: a randomized clinical trial. J. Pediatr. 142, 669-677 (2003).

22. Chmielewska, A. et al. Effects of prenatal and/or postnatal supplementation with iron, PUFA or folic acid on neurodevelopment: update. Br. J. Nutr. 22, 1-6 (2016).

23. Kuipers, R. S. et al. Fetal intrauterine whole body linoleic, arachidonic and docosahexaenoic acid contents and accretion rates. Prostaglandins Leukot. Essent. Fat. Acids 86, 13-20 (2012)

24. Darling, A. L. et al. Association between maternal vitamin D status in pregnancy and neurodevelopmental outcomes in childhood: results from the Avon Longitudinal Study of Parents and Children (ALSPAC). Br. J. Nutr. 117, 1682-1692 (2017).

25. Hayton, S. M., Kriss, A., Wade, A. \& Muller, D. P. The effects of different levels of allrac- and RRR-alpha-tocopheryl acetate (vitamin E) on visual function in rats. Clin. Neurophysiol. 114, 2124-2131 (2003).

26. Hadley, K. B., Ryan, A. S., Forsyth, S., Gautier, S. \& Salem, N. Jr. The essentiality of arachidonic acid in infant development. Nutrients 12, 8 (2016). 216.

27. Lauritzen, L., Fewtrell, M. \& Agostoni, C. Dietary arachidonic acid in perinatal nutrition: a commentary. Pediatr. Res. 77, 263-269 (2015).

28. Brei, C. et al. Impact of the n-6:n-3 long-chain PUFA ratio during pregnancy and lactation on offspring neurodevelopment: 5-year follow-up of a randomized controlled trial. Eur. J. Clin. Nutr. 71, 1114-1120 (2017).

29. Smithers, L. G., Gibson, R. A. \& Makrides, M. Maternal supplementation with docosahexaenoic acid during pregnancy does not affect early visual development in the infant: a randomized controlled trial. Am. J. Clin. Nutr. 93, 1293-1299 (2011).

30. Rangel-Huerta, O. D. \& Gil, A. Effect of omega-3 fatty acids on cognition: an updated systematic review of randomized clinical trials. Nutr. Rev. 76, 1-20 (2018).
31. Shulkin, M. et al. n-3 Fatty acid supplementation in mothers, preterm infants, and term infants and childhood psychomotor and visual development: a systematic review and meta-analysis. J. Nutr. 148, 409-418 (2018).

32. Mendez, M. A. et al. Maternal fish and other seafood intakes during pregnancy and child neurodevelopment at age 4 years. Public Health Nutr. 12, 1702-1710 (2009).

33. Makrides, M., Neumann, M. A., Jeffrey, B., Lien, E. L. \& Gibson, R. A. A randomized trial of different ratios of linoleic to alpha-linolenic acid in the diet of term infants: effects on visual function and growth. Am. J. Clin. Nutr. 71, 120-129 (2000).

34. Judge, M. P., Casavant, S. G., Dias, J. A. \& McGrath, J. M. Reduced DHA transfer in diabetic pregnancies: mechanistic basis and long-term neurodevelopmental implications. Nutr. Rev. 74, 411-420 (2016).

35. Gil-Sánchez, A. et al. Maternal-fetal in vivo transfer of [13 C]docosahexaenoic and other fatty acids across the human placenta $12 \mathrm{~h}$ after maternal oral intake. Am. J. Clin. Nutr. 92, 115-122 (2010).

36. Yeates, A. J. et al. Genetic variation in FADS genes is associated with maternal long-chain PUFA status but not with cognitive development of infants in a high fish-eating observational study. Prostaglandins Leukot. Essent. Fat. Acids 102-103, 13-20 (2015).

37. Bernard, J. Y. et al. EDEN Mother-Child Cohort Study Group The dietary n6:n3 fatty acid ratio during pregnancy is inversely associated with child neurodevelopment in the EDEN mother-child cohort. J. Nutr. 143, 1481-1488 (2013).

38. Makrides, M., Neumann, M. A. \& Gibson, R. A. Perinatal characteristics may influence the outcome of visual acuity. Lipids 36, 897-900 (2001).

39. Campoy, C., Escolano-Margarit, M. V., Anjos, T., Szajewska, H. \& Uauy, R. Omega 3 fatty acids on child growth, visual acuity and neurodevelopment. Br. J. Nutr. 107, S85-S106 (2012).

40. Hautero, U., Poussa, T. \& Laitinen, K. Simple dietary criteria to improve serum n-3 fatty acid levels of mothers and their infants one month after delivery. Public Health Nutr. 20, 534-541 (2017).

41. Ilmonen, J., Isolauri, E. \& Laitinen, K. Nutrition education and counselling practices in mother and child health clinics: study among nurses. J. Clin. Nurs. 21, 2985-2994 (2012). 